\title{
Evaluation of Early Postoperative Intraocular Pressure for Success after Ex-Press Surgery
}

\author{
Naoki Tojo ${ }^{1}$, Atsushi Hayashi ${ }^{2}$, Mitsuya Otsuka ${ }^{3}$
}

\begin{abstract}
Aim: The aim of this study is to identify target levels of early postoperative intraocular pressure (IOP) associated with successful trabeculectomy using an Ex-Press glaucoma shunt.

Materials and methods: This was a retrospective single-facility study. We enrolled 158 glaucoma patients who underwent trabeculectomy with Ex-Press and were followed for $>1$ year, and investigated risk factors for the failure of Ex-Press surgery. We examined age, sex, central corneal thickness (CCT), number of preoperative glaucoma medications, simultaneous performance of cataract surgery, history of trabeculotomy, hypertension (HT), diabetes mellitus (DM), subtype of glaucoma, and early postoperative IOP (minimum, 2 weeks, 1 month, and 3 months).

Results: Ex-Press surgery could significantly decrease IOP. Success rates at 1, 2, 3, and 4 years were 91.1, 86.1, 82.5, and 78.1\%, respectively. Factors significantly affecting the success rate included age, the number of preoperative glaucoma medications, and early postoperative IOP. The IOP cutoff values of minimum IOP for the success of Ex-Press surgery was $5 \mathrm{~mm} \mathrm{Hg}$.

Conclusions: Younger age, a high number of preoperative glaucoma medications, and high IOPs in the early postoperative period were found to be the risk factors for failure of Ex-Press surgery. Considering hypotonic complications, it is desirable to control the minimum IOP from 3-5 $\mathrm{mm} \mathrm{Hg}$ within 2 weeks after surgery. According to our calculations, target IOPs at 2 weeks, 1 month, and 3 months after Ex-Press surgery should be $8 \mathrm{~mm} \mathrm{Hg}, 10 \mathrm{~mm} \mathrm{Hg}$, and $14 \mathrm{~mm} \mathrm{Hg}$, respectively.

Clinical significance: We thought that Ex-Press surgery might require lower IOP in the early postoperative period than conventional trabeculectomy.

Keywords: Choroidal detachment, Ex-Press, Target intraocular pressure, Trabeculectomy.

Journal of Current Glaucoma Practice (2019): 10.5005/jp-journals-10078-1252
\end{abstract}

\section{INTRODUCTION}

Trabeculectomy is a surgical filtering method that is widely used in the treatment of glaucoma. In recent years, trabeculectomies using an Ex-Press ${ }^{\circledast}$ Mini Glaucoma Shunt Device (hereafter, "Ex-Press"; Alcon Laboratories, Fort Worth, TX, USA) have been conducted worldwide. ${ }^{1}$ The Ex-Press is a stainless-steel filtration device designed to shunt the aqueous humor from the anterior chamber to the subconjunctival space. In a trabeculectomy with an Ex-Press, there is no need to remove the trabecular meshwork and the peripheral iris; thus, this technique is less invasive than a conventional trabeculectomy.

IOP control at the early postoperative period has been shown to be critical for successful trabeculectomy. ${ }^{2-4}$ It is no exaggeration to say that long-term surgical results are determined by early IOP control. If early postoperative IOP is too low, complications such as choroidal detachment (CD), shallow anterior chamber, and hypotony maculopathy can occur; if it is too high, surgery is unsuccessful. Several studies on target IOP in the early postoperative period after conventional trabeculectomy have been reported, but there have been no such reports for Ex-Press surgery. ${ }^{5-7}$ Some studies have reported that trabeculectomy with Ex-Press has less risk of hypotony because the amount of aqueous humor exiting the bleb from the anterior chamber is limited. ${ }^{8-10}$ It has not been evaluated whether the target IOP of Ex-Press surgery should be the same as that of conventional trabeculectomy.

The IOP can be expected to increase as time elapses after Ex-Press surgery. The question is, How low should the IOP go immediately after surgery? We investigated early postoperative
${ }^{1-3}$ Department of Ophthalmology, Graduate School of Medicine and Pharmaceutical Sciences, University of Toyama, Toyama, Japan

Corresponding Author: Naoki Tojo, Department of Ophthalmology, Graduate School of Medicine and Pharmaceutical Sciences, University of Toyama, Toyama, Japan, Phone: +81-76-434-7363, e-mail: tojonaoki@umin.ac.jp

How to cite this article: Tojo N, Hayashi A, et al. Evaluation of Early Postoperative Intraocular Pressure for Success after Ex-Press Surgery. J Curr Glaucoma Pract 2019;13(2):55-61.

Source of support: Nil

Conflict of interest: None

IOPs associated with success. We also examined the risk factors for Ex-Press surgery failure.

\section{Materials and Methods Subjects}

This was a retrospective, nonrandomized observational study. We analyzed the cases of 158 consecutive patients (191 eyes) who underwent trabeculectomy with Ex-Press for the first time at Toyama University Hospital and were followed for $>1$ year. All patients were Japanese. There were 33 patients who underwent trabeculectomy in both eyes, and in these cases, we used only unilateral data of the eye that was operated first. All subjects were recruited during the period from January 2013 to May 2017. All patients underwent a comprehensive ophthalmic examination including refraction, Goldmann gonioscopy, Goldmann applanation

() The Author(s). 20190pen Access This article is distributed under the terms of the Creative Commons Attribution 4.0 International License (https://creativecommons. org/licenses/by-nc/4.0/), which permits unrestricted use, distribution, and non-commercial reproduction in any medium, provided you give appropriate credit to the original author(s) and the source, provide a link to the Creative Commons license, and indicate if changes were made. The Creative Commons Public Domain Dedication waiver (http://creativecommons.org/publicdomain/zero/1.0/) applies to the data made available in this article, unless otherwise stated. 
tonometry (GAT), fundus examination, automated perimetry (Humphrey Field Analyzer; Carl Zeiss Meditec, Dublin, CA), and measurement of CCT and anterior chamber depth with anterior segment optical coherence tomography (CASIA SS-1000; Tomey, Nagoya, Japan). Two glaucoma specialists (NT and AH) diagnosed all glaucoma cases.

The patients had already used tolerated glaucoma medications, and they had needed further treatment to lower their IOP because of progression of their visual field disorder. The research protocol was approved by the Institutional Review Board of the University of Toyama, and the procedures used conformed to the tenets of the Declaration of Helsinki. After the nature and possible consequences of the study were explained to the patients, written informed consent was obtained from each patient.

The preoperative (baseline) IOP was the mean of the IOPs recorded at three of the patient's visits while on preoperative treatment. The IOP was measured using GAT.

\section{Surgical Techniques}

Surgery in all patients was performed by the same surgeon (N.T.). Retrobulbar anesthesia was administered. A standard fornix-based conjunctival incision was made to gain exposure to the scleral bed adjacent to the limbus. A single $3.5 \mathrm{~mm}^{2}$ square scleral flap was created. Mitomycin C (MMC) solution $(0.04 \mathrm{mg} / \mathrm{mL})$ was applied for 4 minutes. At this point, the eye was a completely enclosed space; thus, the MMC solution could not flow into the anterior chamber. The treated area was then irrigated with approx. $100 \mathrm{~mL}$ of balanced salt solution. If the patient needed simultaneous cataract surgery, the cataract surgery was performed at this time. Regarding surgical indications for cataract surgery, our study is retrospective and no clear criteria had been established; hence, cataract surgery was performed according to the judgment of the operator.

To create a path for the Ex-Press ${ }^{\circledast}$ (model P50), the scleral flap was lifted, and a 25-gauge needle was horizontally inserted into the anterior chamber at the surgical limbus from the sclera-cornea transition zone parallel with the iris. The Ex-Press was then inserted into the anterior chamber.

The scleral flap was sutured using a 10-0 nylon suture, and the tension of the sutures was adjusted to maintain anterior chamber depth with a slow flow of aqueous humor around the margins of the scleral flap. The conjunctiva was meticulously closed with 10-0 nylon suture. We confirmed that there was no leakage from the blebs.

\section{Postoperative Medication}

The postoperative treatments were topical medications of steroids, antibiotics, and nonsteroidal anti-inflammatory drugs (NSAIDs). The antibiotics were used four times per day for 4 weeks after surgery. Steroids were administered eight times in the hospital. After discharge from the hospital (typically at 1 week), the steroid dose was reduced over 12 weeks. NSAIDs were used two times per day for 12 weeks. Antiglaucoma medications were added the discretion of the physicians. We counted a compounding agent as two medications.

\section{Factors for Failure}

We conducted a statistical analysis to determine whether various factors influenced the result of the Ex-Press surgery. These factors were age, sex, CCT, preoperative IOP, number of preoperative glaucoma medications, single vs triple surgery, (Ex-Press surgery alone or phacoemulsification + simultaneous intraocular lens implantation and Ex-Press surgery, respectively), history of trabeculotomy (including Trabectome ${ }^{\circledR}$ and canaloplasty) HT, DM, subtype of glaucoma (primary open-angle glaucoma (POAG) or pseudo exfoliation glaucoma (PEXG)), and postoperative IOPs at early time points. Regarding HT and DM, we only confirmed by inquiry; we did not actually take blood samples or measure blood pressure. We examined early postoperative IOP by four values: minimum IOP and IOP at 2 weeks, 1 month, and 3 months. We defined minimum IOP as the lowest IOP value within 14 days after surgery with or without postoperative interventions (laser suture-lysis or needling).

\section{Definition of Success}

As the main criteria of successful treatment, we used postoperative IOP $\leq 21 \mathrm{~mm} \mathrm{Hg}$ or a $\geq 20 \%$ reduction from baseline IOP on two consecutive visits after the 1st postoperative month. Cases were considered treatment failures if neither of the success criteria were met at two consecutive visits after the first postoperative month. Eyes requiring additional glaucoma surgery and those that developed phthisis or showed loss of light perception were classified as failures. Subjects who underwent additional surgery after achieving $\geq 20 \%$ IOP reduction from the baseline were counted as "additional surgery."

\section{Statistical Analysis}

A paired $t$ test was used for comparison between the groups. A Wilcoxon signed-rank test was used for the comparison of the same patients of IOP, the number of glaucoma medications, and visual acuity (VA). Kaplan-Meier survival analysis and log-rank tests were used for the comparison of the success rate. Risk factors for failure were identified using logistic regression analysis and multiple regression analysis. The IOP cutoff value for success was calculated from the receiver operating characteristic (ROC) curve. All statistical analyses were performed with JMP Pro 11 software (SAS, Cary, NC). The significance was defined as $p$ values $<0.05$.

\section{Results \\ Ophthalmic Data}

The characteristics of two groups are shown in Table 1. We analyzed the cases of 158 patients, including 92 males and 66 females. The mean ( \pm standard deviation) values for all 158 patients are as follows: age at the time of surgery, $70.8 \pm 10.3$ years; CCT, $529 \pm 34 \mu \mathrm{m}$; follow-up period, $28.1 \pm 15.3$ months; number of glaucoma medications, $4.0 \pm 1.0 \mathrm{drops}$; and preoperative IOP, $24.5 \pm 9.0 \mathrm{~mm} \mathrm{Hg}$. Fifty patients with a phakic eyes underwent cataract surgery and a trabeculectomy with Ex-Press at the same surgery session. Thirty-four patients had a history of trabeculotomy (metal-probe trabeculotomy, 15 eyes; canaloplasty, 2 eyes; trabeculotomy with Trabectome ${ }^{\circledR}, 17$ eyes).POAG was present in 70 eyes, PEXG in 85 eyes, and secondary glaucoma (SG) in 3 eyes. Since Ex-Press is contraindicated for use in patients with uveitis or primary angle-closure glaucoma (PACG), there were a few SG patients and no case of PACG.

\section{Postoperative IOP, Number of Medications Used and Success Rate}

Table 2 summarizes the postoperative data of IOP and the number of glaucoma medications. The results of the eyes that underwent additional glaucoma surgeries were excluded. The mean postoperative IOP and the mean number of glaucoma medications were significantly decreased at all time points. The mean number of glaucoma medications gradually increased after Ex-Press surgery. 
Table 1: Ophthalmic data

\begin{tabular}{ll}
\hline No. of eyes & Mean \pm SD \\
\hline Age (years) & $70.8 \pm 10.3$ \\
Gender (male/female) & $92 / 66$ \\
Follow-up (months) & $28.1 \pm 15.3$ \\
CCT $(\mu \mathrm{m})$ & $529 \pm 34$ \\
Pre-IOP (mm Hg) & $24.5 \pm 9.0$ \\
No. of premedications & $4.0 \pm 1.0$ \\
Triple surgery & $50 / 158(31.6 \%)$ \\
History of TLO & $34 / 158(21.5 \%)$ \\
HT & $73 / 158(46.2 \%)$ \\
DM & $32 / 158(20.2 \%)$ \\
Subtype of glaucoma & \\
POAG & $70 / 158(44.3 \%)$ \\
PEXG & $85 / 158(53.8 \%)$ \\
SG & $3 / 158(1.9 \%)$ \\
\hline
\end{tabular}

SD, standard deviation; Triple surgery, trabeculectomy and phacoemulsification + intraocular lens implantation simultaneously; TLO, trabeculotomy (including trabectome, canaloplasty); POAG, primary open-angle glaucoma; PEX, pseudo-exfoliation; SG, secondary glaucoma

Table 2: The results of postoperative IOP and number of medications

\begin{tabular}{lcc}
\hline Period $(n)$ & $I O P(\mathrm{~mm} \mathrm{Hg})$ & Medications \\
\hline Minimum (158) & $3.8 \pm 2.4$ & $0 \pm 0$ \\
2 weeks (158) & $5.2 \pm 4.6$ & $0 \pm 0$ \\
1 month (158) & $9.7 \pm 4.9$ & $0 \pm 0$ \\
3 months (158) & $11.7 \pm 5.2$ & $0.5 \pm 1.2$ \\
12 months (144) & $11.1 \pm 3.5$ & $1.4 \pm 1.6$ \\
24 months (84) & $11.8 \pm 3.8$ & $1.9 \pm 1.7$ \\
36 months (41) & $11.6 \pm 4.5$ & $2.5 \pm 1.6$ \\
48 months (19) & $11.9 \pm 3.8$ & $2.4 \pm 1.6$ \\
60 months (5) & $11.8 \pm 1.7$ & $3.0 \pm 1.7$ \\
\hline
\end{tabular}

Numbers in parentheses indicate the number of eyes

We performed a Kaplan-Meier analysis to determine the success rates of Ex-Press surgery (Fig. 1). Surgery failed in 22 of 158 eyes (13.9\%). The reasons for failure were as follows: need for additional glaucoma surgery, 22 eyes; IOP reduction rate less than $20 \%$, 4 eyes; and loss of light sensation, 2 eyes.

\section{Analysis of the Factors of Failure}

Results of the factors-of-failure analysis are shown in Table 3. Among the items, significant differences were found for age ( $p=0.0028)$, number of pre-operative medications ( $p=0.0143$ ), postoperative minimum IOP ( $p=0.0004)$, IOP at 2 weeks after surgery $(p=0.0097)$ IOP at 1 month after surgery $(p=0.0118)$, and IOP at 3 months after surgery $(p<0.0001)$. The upper cutoff value of minimum IOP for success was $5 \mathrm{~mm} \mathrm{Hg}$. Similarly, the cutoff value of IOP after 2 weeks, 1 month, and 3 months were $8 \mathrm{~mm} \mathrm{Hg}, 10 \mathrm{~mm} \mathrm{Hg}$, and $14 \mathrm{~mm} \mathrm{Hg}$, respectively (Table 4). We divided by each cutoff value and compared with the Kaplan-Meier analysis in Figure 2.

\section{Complications}

Postoperative complications are summarized in Table 5. Postoperative choroidal detachment (CD) was defined as a solidappearing elevation of the retina and choroid with fundoscopic examination. CD causing hypotony was identified in 40 eyes (25.3\%). In all cases, CD disappeared within 2 months.

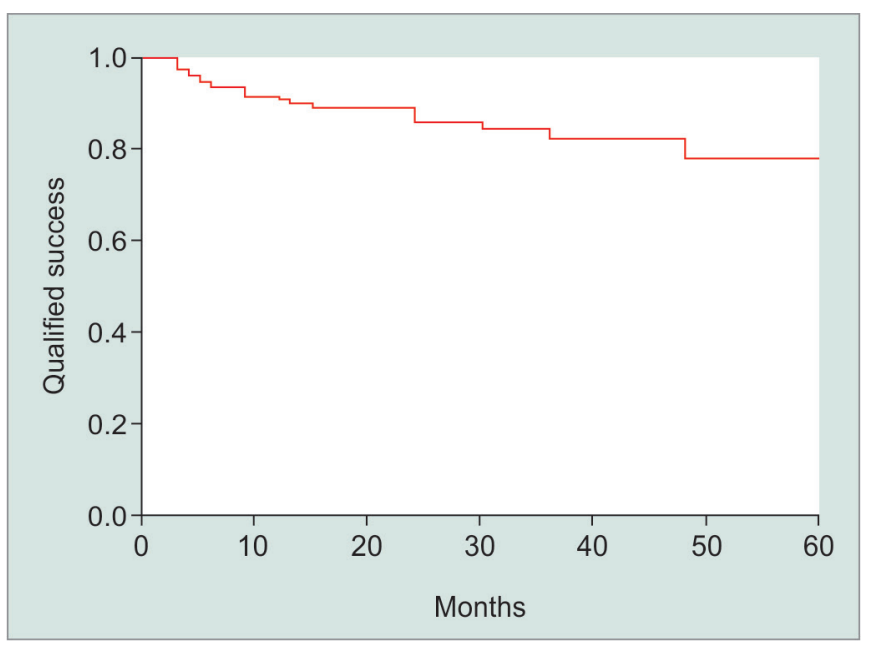

Fig. 1: Kaplan-Meier survival plots of all cases for qualified success. The success was defined as postoperative IOP $\leq 21 \mathrm{~mm} \mathrm{Hg}$, at least $20 \%$ IOP reduction from baseline in any two consecutive visits after 3 months, and no secondary glaucoma or lens surgery

A shallow anterior chamber was clinically assessed under slit-lamp examination and was defined as iridocorneal touch in the periphery. A shallow anterior chamber occurred in nine cases (5.7\%). In all cases, the shallow anterior chamber was improved by suturing the scleral flap or spontaneously within 1 month. The cutoff value of the minimum IOP for CD or shallow anterior chamber was $2 \mathrm{~mm} \mathrm{Hg}$ (Table 4).

Hyphema was considered present when pooling of blood was seen in the anterior chamber. Hyphema occurred in six cases (3.8\%). In all cases of hyphema, bleeding in the anterior chamber disappeared spontaneously. There were no cases requiring additional surgery among those with $C D$, shallow anterior chamber or hyphema.

Two cases resulted in bullous keratopathy. In one case, Descemet's membrane endothelial keratoplasty was performed,

Table 3: Analysis of risk factors for failure of Ex-Press surgery

\begin{tabular}{lllll}
\hline Factors & Odds ratio & $95 \%$ lower Cl & $95 \%$ upper Cl & p value \\
\hline Age & 0.938 & 0.898 & 0.978 & 0.0028 \\
Gender & 1.042 & 0.421 & 2.682 & 0.929 \\
CCT & 1.01 & 0.997 & 1.024 & 0.123 \\
Pre-IOP & 1.041 & 0.992 & 0.96 & 0.0984 \\
No. of & 1.622 & 1.019 & 2.622 & 0.0143 \\
premedications & & & & \\
Triple surgery & 1.644 & 0.633 & 4.124 & 0.299 \\
History of TLO & 0.785 & 0.214 & 2.298 & 0.676 \\
HT & 0.756 & 0.294 & 1.871 & 0.547 \\
DM & 0.353 & 0.0545 & 1.309 & 0.129 \\
Subtype of & 1.747 & 0.693 & 4.458 & 0.237 \\
glaucoma & & & & \\
Minimum IOP & 1.366 & 1.143 & 1.679 & 0.0004 \\
Post 2 W IOP & 1.146 & 1.035 & 1.278 & 0.0097 \\
Post 1 M IOP & 1.104 & 1.078 & 1.198 & 0.0118 \\
Post 3 M IOP & 1.196 & 1.099 & 1.322 & $<0.0001$ \\
\hline
\end{tabular}

$\mathrm{Cl}$, confidence interval; Triple surgery, trabeculectomy and phacoemulsification + intraocular lens implantation simultaneously; TLO, trabeculotomy (including trabectome, canaloplasty); $\mathrm{W}$, week; $\mathrm{M}$, month 
Table 4: The cutoff values of postoperative IOP

\begin{tabular}{|c|c|c|c|c|}
\hline Factors & AUC & Sensitivity & 1-Specificity & $\begin{array}{l}\text { Cutofflevel } \\
(\mathrm{mm} \mathrm{Hg})\end{array}$ \\
\hline $\begin{array}{l}\text { Choroidal } \\
\text { Detachment }\end{array}$ & 0.798 & 0.675 & 0.17 & 2 \\
\hline $\begin{array}{l}\text { Shallow anterior } \\
\text { chamber }\end{array}$ & 0.775 & 0.778 & 0.269 & 2 \\
\hline Minimum IOP & 0.642 & 0.853 & 0.591 & 5 \\
\hline Post 2 weeks IOP & 0.631 & 0.636 & 0.912 & 8 \\
\hline Post 1 month IOP & 0.661 & 0.691 & 0.455 & 10 \\
\hline Post 3 months IOP & 0.763 & 0.824 & 0.421 & 14 \\
\hline
\end{tabular}

AUC, area under the curve

and in another case, corneal transplantation was not desired. One case each of expulsive hemorrhage and endophthalmitis occurred, and vitrectomy was performed. There was no case of hypotony maculopathy.
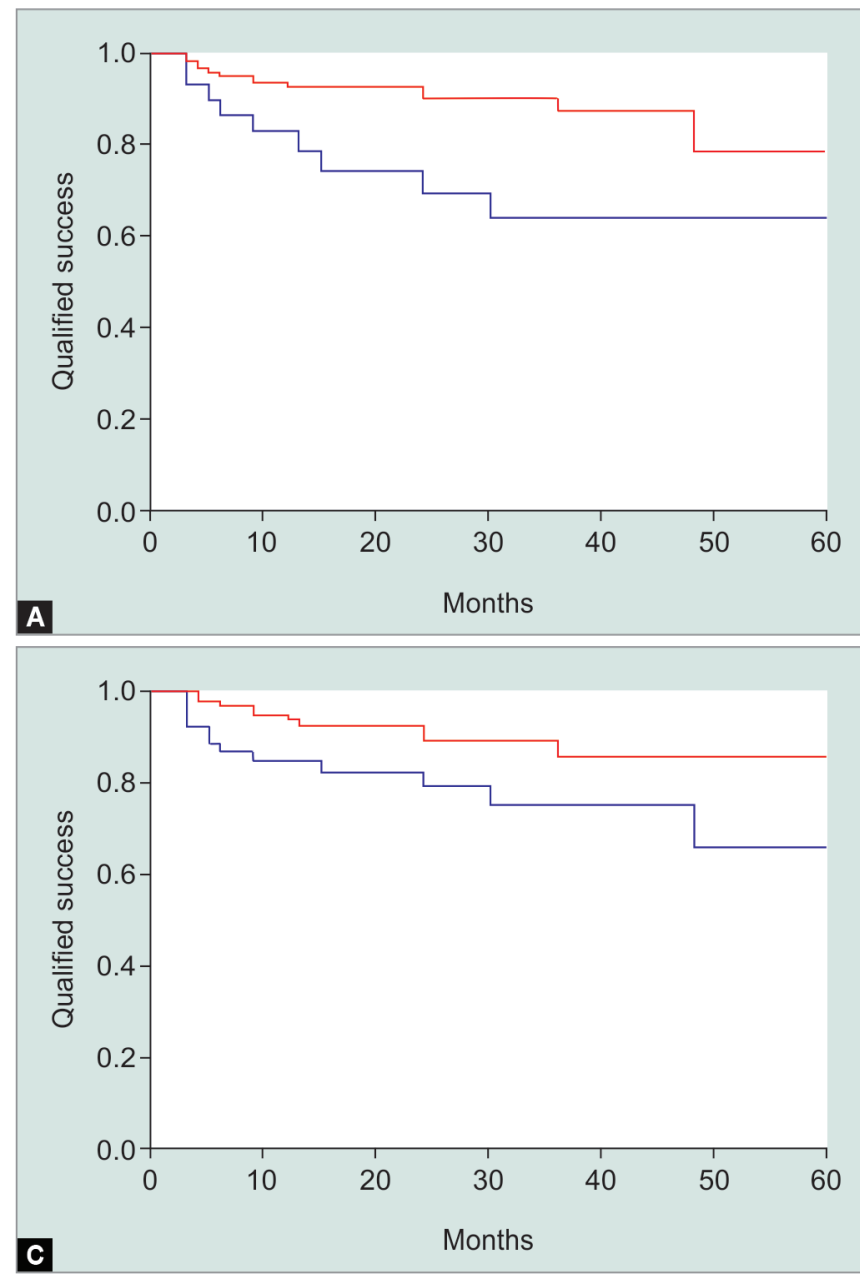

B

\section{Discussion}

Ex-Press surgery could significantly decrease IOP at all time points $(p<0.0001)$. The success rate was $91.1,86.1,82.5,78.1$, and $78.1 \%$ after $1,2,3,4$, and 5 years, respectively. The results showed that younger age, high number of preoperative glaucoma medications and high early postoperative IOP were risk factors for failure of Ex-Press surgery. Our success rate and postoperative IOP values were similar to those previously described while the number of postoperative glaucoma medications tended to be somewhat higher. ${ }^{9,11-14}$

Several previous studies have examined risk factors in conventional trabeculectomy; they reported that age, preoperative IOP, number of preoperative glaucoma medications, subtype of glaucoma, triple surgery, DM, and postoperative complications are risk factors. ${ }^{15-17}$ Mariotti et al. reported that identifiable risk factors for failure of Ex-Press surgery are DM and previous glaucoma surgery. ${ }^{11}$ In our study, DM and previous glaucoma surgery did not significantly influence Ex-Press surgery outcomes. Ochiai et al. reported that transforming growth factor (TGF) $\beta 2$ concentration
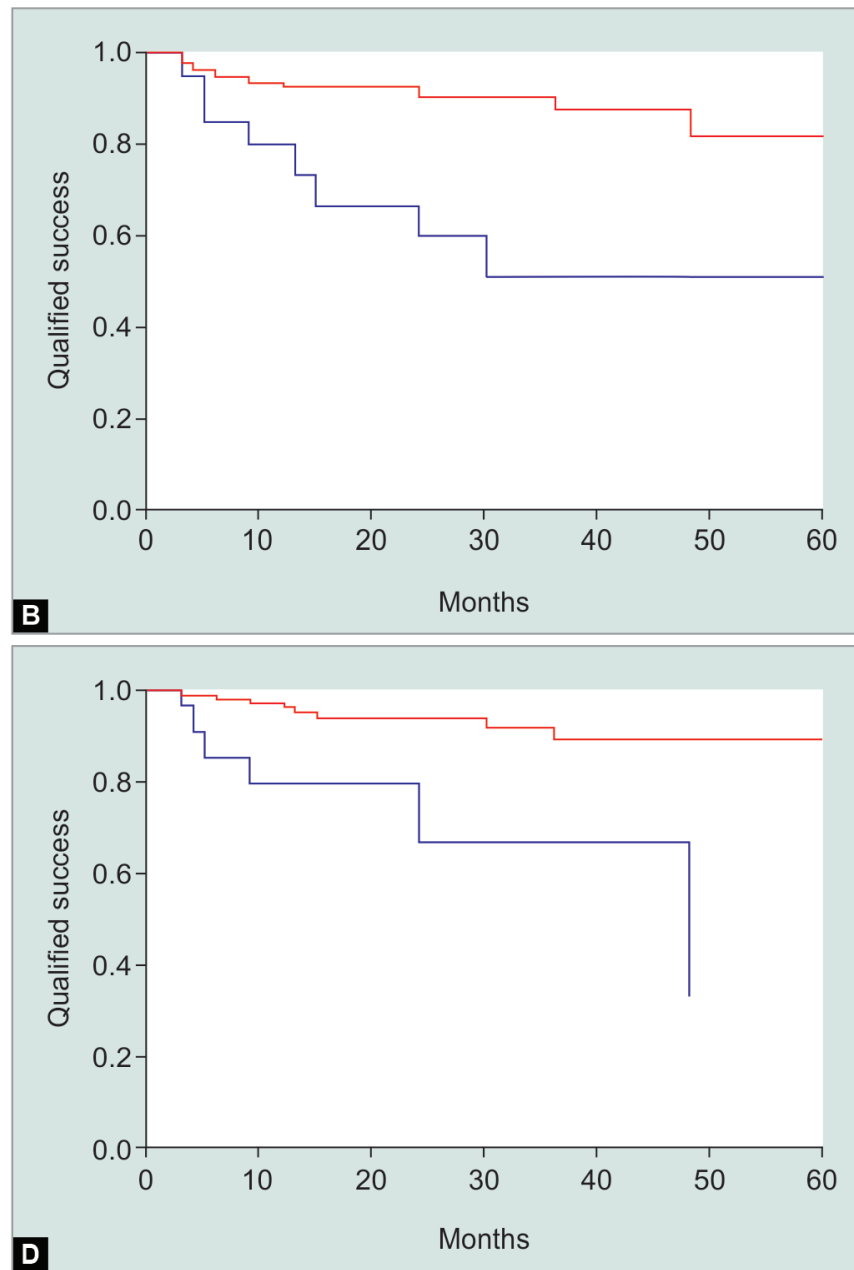

Figs $2 \mathrm{~A}$ to D: (A) Kaplan-Meier survival plots comparing minimum IOP $\leq 5 \mathrm{~mm} \mathrm{Hg}$ (128 eyes) and minimum IOP $>6 \mathrm{~mm} \mathrm{Hg}$ (30 eyes) within 2 weeks after Ex-Press surgery. The group of postoperative IOP $\leq 5 \mathrm{~mm} \mathrm{Hg}$ (bold line) was significantly better than the group of postoperative IOP $>6 \mathrm{~mm} \mathrm{Hg}$ (normal line) $(p=0.0118$ ); (B) Kaplan-Meier survival plots comparing postoperative IOP $\leq 8 \mathrm{~mm} \mathrm{Hg}$ (138 eyes) and postoperative IOP $>9 \mathrm{~mm} \mathrm{Hg}$ (20 eyes) at 2 weeks after Ex-Press surgery. The group of postoperative IOP $\leq 8 \mathrm{~mm} \mathrm{Hg}$ (bold line) was significantly better than the group of postoperative IOP $>9 \mathrm{~mm} \mathrm{Hg}$ (normal line) $(p=0.0006)$; (C) Kaplan-Meier survival plots comparing postoperative IOP $\leq 10 \mathrm{~mm}$ Hg (104 eyes) and postoperative IOP $>11 \mathrm{~mm} \mathrm{Hg}$ (54 eyes) at 1 month after Ex-Press surgery. The group of postoperative IOP $\leq 10 \mathrm{~mm} \mathrm{Hg}$ (bold line) was significantly better than the group of postoperative IOP $>11 \mathrm{~mm} \mathrm{Hg}$ (normal line) $(p=0.0354$ ); (D) Kaplan-Meier survival plots comparing postoperative IOP $\leq 14 \mathrm{~mm} \mathrm{Hg}$ (120 eyes) and postoperative IOP $>15 \mathrm{~mm} \mathrm{Hg}$ (35 eyes) at 3 months after Ex-Press surgery. The group of postoperative $\mathrm{IOP} \leq 14 \mathrm{~mm} \mathrm{Hg}$ (bold line) was significantly better than the group of postoperative IOP $>15 \mathrm{~mm} \mathrm{Hg}$ (normal line) $(p<0.0001)$ 
Table 5: Complications

\begin{tabular}{ll}
\hline Complications & Ratio (\%) \\
\hline Choroidal detachment & $40 / 158(25.3)$ \\
Shallow anterior chamber & $9 / 158(5.7)$ \\
Hyphema & $6 / 158(3.8)$ \\
Bullous keratopathy & $2 / 158(1.3)$ \\
Expulsive hemorrhage & $1 / 158(0.6)$ \\
Endophthalmitis & $1 / 158(0.6)$ \\
Hypotony maculopathy & $0 / 158(0)$ \\
\hline
\end{tabular}

influences the progression of POAG. ${ }^{18}$ Min et al. reported that level of TGF- $\beta 2$ are high in diabetic patients and that TGF- $\beta 2$ may play a role in the pathology of glaucoma. ${ }^{19}$ The absence of a medical DM check might affect the surgical outcome. Previous glaucoma surgery involves ab intero surgery (without conjunctival incision) and ab externo surgery (with conjunctival incision). The surgical outcome may depend on whether conjunctival incision is involved. In our study, results were not significantly different between previous glaucoma surgery with conjunctival incision and those without conjunctival incision ( $p=0.277$ ); however, the number of cases might have been too small for meaningful analysis.

Regarding conventional trabeculectomy, many previous epidemiologic studies have reported that younger age was a risk factor of failure. ${ }^{3,16}$ One reason for this finding is considered to be the more vigorous healing response resulting in a thicker Tenon's capsule. $^{20}$

PEXG has been associated with a risk of trabeculectomy failure, especially over the long-term. ${ }^{15,21}$ Djordjević-Jocić et al. reported that PEXG is associated with inflammatory cytokines that might reduce the volume of blebs. ${ }^{22}$ In our study, there was no significant difference between POAG and PEXG. We need to evaluate further long-term results.

It was reported that a larger number of preoperative glaucoma medications constituted a risk for failure of conventional trabeculectomy. ${ }^{15}$ Sherwood et al. reported that the use of longterm glaucoma medications affected the conjunctiva and Tenon's capsule. $^{23}$

It was also reported that triple surgery was a risk factor for failure of conventional trabeculectomy. ${ }^{17}$ Takihara et al. reported that intraocular surgery might cause ocular inflammation and breakdown of the blood-aqueous barrier, which may cause bleb failure after trabeculectomy. Monocyte chemoattractant protein 1, which arises after phacoemulsification, might promote subconjunctival fibrosis and bleb scarring after triple surgery. ${ }^{24}$

Many previous studies have reported that lowering the IOP at an early postoperative period was an important factor for the success of trabeculectomy. To sufficiently lower the IOP at the early postoperative period, early postoperative interventions such as laser suture lysis are necessary. ${ }^{4,25,26}$ The timing of laser suture lysis is very important for trabeculectomy; there are reports that laser suture lysis within $7 \pm 10$ days lowers postoperative IOP. ${ }^{427-29}$ We previously reported that the mean period before undergoing laser suture lysis of the first thread after Ex-Press surgery was 3.4 days. ${ }^{30}$ The timing of laser suture lysis of the first thread in Ex-Press surgery is significantly sooner than that in conventional trabeculectomy. ${ }^{9,30}$

Okamoto et al. reported that IOP under $8 \mathrm{~mm} \mathrm{Hg}$ at 2 weeks after conventional trabeculectomy was associated with maintaining the postoperative IOP at either $\leq 11$ and $\leq 15 \mathrm{~mm} \mathrm{Hg}$ for an extended period. $^{3}$ In our study, the mean postoperative IOP at 2 weeks after Ex-Press surgery was $5.2 \pm 4.6 \mathrm{~mm} \mathrm{Hg}$. The IOP cutoff value at 2 weeks for successful Ex-Press was $8 \mathrm{~mm} \mathrm{Hg}$. Lowering the minimum IOP to $5 \mathrm{~mm} \mathrm{Hg}$ or less improved results of Ex-Press surgery. This does not state that it is better to cut threads or to do needling when pressure exceeds $5 \mathrm{~mm} \mathrm{Hg}$. However, our results do suggest that laser suture lysis or needling aiming at $5 \mathrm{~mm} \mathrm{Hg}$ or less after surgery may lead to better results.

Generally, the value of $5 \mathrm{~mm} \mathrm{Hg}$ is considered too low. We must be careful that complications may occur due to lowering IOP. In our study, there were 40 cases in which CD occurred, and the cutoff value for CD occurring was $2 \mathrm{~mm} \mathrm{Hg}$. Tanihara et al. reported that CD occurs at $5.5 \mathrm{~mm} \mathrm{Hg}$ in conventional trabeculectomy. In previous studies, $11-24.3 \%$ of patients who underwent conventional trabeculectomy developed CD. ${ }^{31-33}$ The frequency of CD in Ex-Press surgery is reported to be $3.8 \pm 7.5 \%$, less than that of conventional trabeculectomy. $9,10,13$ This might suggest that Ex-Press surgery can reduce IOP lower than conventional trabeculectomy without hypotonic complications. Our result that $25.3 \%$ of patients undergoing Ex-Press surgery developed CD is higher than previous reports. This result might be due to more aggressive laser suture lysis than in previous reports. Altan et al. reported that the presence or absence of CD does not affect the postoperative VA or IOP. In our study, there was no significant difference in surgical results ( $p=0.393$ ) or VA change ( $p=0.720)$ between patients with and without CD. In all cases in which it developed, CD disappeared within 2 months after surgery. If the only complication after Ex-Press surgery is $C D$, it might not be that big a problem.

Reducing IOP too much could cause various complications such as shallow anterior chamber and hypotony maculopathy in addition to $C D$. A shallow anterior chamber can cause a rapid decrease in corneal endothelial cells. ${ }^{34}$ Hypotony is also a risk factor for loss of visual field for patients with severe glaucoma. ${ }^{35,36}$ Benson et al. reported that early post-trabeculectomy hypotony (within 1 month) is associated with reduced survival time of blebs. ${ }^{37}$ Laser suture lysis is necessary for lowering IOP, but we need to take care not to cause hypotony. ${ }^{38,39}$ In fact, the timing of laser suture lysis varies depending on each case.

Laser suture lysis is difficult to perform in cases of leaking blebs in the early postoperative period, and this may increase the risk for surgical failure. A leaking bleb at an early postoperative period was considered an indication for intervention such as conjunctival suture within 2 weeks after Ex-Press surgery. In our study, there were 14 cases of leaking bleb (8.9\%). Leaking bleb was not a significant risk factor for surgical failure $(p=0.132)$.

Our study has several limitations. The first is its retrospective design with the potential for investigator bias in the selection of cases and loss to follow-up. The losses to follow-up were realistic and were taken into account by the statistical analysis. Because this was a large consecutive case series with a long follow-up, the risk factors for failure were detected more accurately than in smaller series with shorter follow-up. The second is that our study was shortterm periods for follow-up. The third is that we did not conduct medical checks regarding $\mathrm{HT}$ and DM. The fourth was that our study was the small number of patients. A larger prospective comparative study is needed to fully investigate the issues explored here.

\section{ConCLUSiOn}

The control of postoperative IOP seems to become more difficult as time after surgery elapses. For successful Ex-Press surgery, it is important to reduce IOP in the early postoperative period 
(within 2 weeks) to $5 \mathrm{~mm} \mathrm{Hg}$ or less. Considering hypotonic complications, the target of minimum IOP should be from 3 to $5 \mathrm{~mm} \mathrm{Hg}$. Furthermore, it is desirable that the IOP value at 2 weeks postsurgery not exceed $8 \mathrm{~mm} \mathrm{Hg}$.

\section{Clinical Significance}

We thought that Ex-Press surgery might require lower IOP in the early postoperative period than conventional trabeculectomy.

\section{Ethical Approval}

All procedures performed in studies involving human participants were in accordance with the ethical standards of the institutional and/or national research committee and with the 1964 Helsinki declaration and its later amendments or comparable ethical standards. For this type of study, formal consent is not required.

\section{References}

1. Sarkisian SR. The ex-press mini glaucoma shunt: technique and experience. Middle East Afr J Ophthalmol 2009;16:134-137. DOI: 10.4103/0974-9233.56226.

2. Rong SS, Meng HL, et al. Can intraoperative intraocular pressure during primary trabeculectomy predict early postoperative pressure? J Glaucoma 2014;23:653-657. DOI: 10.1097/IJG.0b013e318285ff44.

3. Okimoto S, Kiuchi Y, et al. Using the early postoperative intraocular pressure to predict pressure control after a trabeculectomy. J Glaucoma 2014;23:410-414. DOI: 10.1097/IJG.0b013e318285fd7d.

4. Asamoto A, Yablonski ME, et al. Predicting long-term results of trabeculectomy from early postoperative intraocular pressure levels. Ophthalmic Surg Lasers 1996;27:355-360.

5. Schultz SK, Iverson SM, et al. Safety And Efficacy Of Achieving SingleDigit Intraocular Pressure Targets With Filtration Surgery In Eyes With Progressive Normal-Tension Glaucoma. J Glaucoma 2016;25:217-222. DOI: $10.1097 /$ IJG.0000000000000145.

6. Aoyama $\mathrm{Y}$, Murata $\mathrm{H}$, et al. Targeting a low-teen intraocular pressure by trabeculectomy with a fornix-based conjunctival flap: continuous Japanese case series by a single surgeon. J Glaucoma 2015;24: 225-232. DOI: 10.1097/IJG.0000000000000219.

7. Iverson SM, Schultz SK, et al. Effectiveness of Single-Digit IOP Targets on Decreasing Global and Localized Visual Field Progression After Filtration Surgery in Eyes With Progressive Normal-Tension Glaucoma. J Glaucoma 2016;25:408-414. DOI: 10.1097/IJG.0000000000000240.

8. Dahan E, Ben Simon GJ, et al. Comparison of trabeculectomy and Ex-PRESS implantation in fellow eyes of the same patient: a prospective, randomised study. Eye 2012;26:703-710. DOI: 10.1038/ eye.2012.13.

9. Good TJ, Kahook MY. Assessment of bleb morphologic features and postoperative outcomes after Ex-PRESS drainage device implantation vs trabeculectomy. Am J Ophthalmol 2011;151:507-513 e501. DOI: 10.1016/j.ajo.2010.09.004.

10. Netland PA, Sarkisian Jr SR, et al. Randomized, prospective, comparative trial of EX-PRESS glaucoma filtration device vs trabeculectomy (XVT study). Am J Ophthalmol 2014;157:433-440 e433. DOI: 10.1016/j.ajo.2013.09.014.

11. Mariotti C, Dahan E, et al. Long-term outcomes and risk factors for failure with the EX-press glaucoma drainage device. Eye 2014;28:1-8. DOI: 10.1038/eye.2013.234.

12. Gonzalez-RodriguezJM, Trope GE, etal.Comparison of trabeculectomy vs Ex-PRESS: 3-year follow-up. Br J Ophthalmol 2016;100:1269-1273. DOI: 10.1136/bjophthalmol-2015-307161.

13. de Jong LA. The Ex-PRESS glaucoma shunt vs trabeculectomy in open-angle glaucoma: a prospective randomized study. Adv Ther 2009;26:336-345. DOI: 10.1007/s12325-009-0017-6.

14. Lankaranian D, Razeghinejad MR, et al. Intermediate-term results of the Ex-PRESS miniature glaucoma implantunder a scleral flap in previously operated eyes. Clin Exp Ophthalmol 2011;39:421-428. DOI: 10.1111/j.1442-9071.2010.02481.x.

15. Landers J, Martin K, et al. A twenty-year follow-up study of trabeculectomy: risk factors and outcomes. Ophthalmology 2012;119:694-702. DOI: 10.1016/j.ophtha.2011.09.043.

16. AGIS Investigators. The Advanced Glaucoma Intervention Study (AGIS): 11. Risk factors for failure of trabeculectomy and argon laser trabeculoplasty. Am J Ophthalmol 2002;134:481-498. DOI: 10.1016/ S0002-9394(02)01658-6.

17. Ogata-Iwao $M$, Inatani $M$, et al. A prospective comparison between trabeculectomy with mitomycin $\mathrm{C}$ and phacotrabeculectomy with mitomycin C. Acta Ophthalmol 2013;91:e500-e501. DOI: 10.1111/ aos.12133.

18. Ochiai $\mathrm{Y}$, Ochiai H. Higher concentration of transforming growth factor-beta in aqueous humor of glaucomatous eyes and diabetic eyes. Jpn J Ophthalmol 2002;46:249-253. DOI: 10.1016/S00215155(01)00523-8.

19. Min SH, Lee Tl, et al. Transforming growth factor-beta levels in human aqueous humor of glaucomatous, diabetic and uveitic eyes. Korean J Ophthalmol 2006;20:162-165. DOI: 10.3341/kjo.2006.20.3.162.

20. Broadway DC, Chang LP. Trabeculectomy, risk factors for failure and the preoperative state of the conjunctiva. J Glaucoma 2001;10:237249. DOI: 10.1097/00061198-200106000-00017.

21. Lim SH, Cha SC. Long-term Outcomes of Mitomycin-C Trabeculectomy in Exfoliative Glaucoma Vs Primary Open-Angle Glaucoma. J Glaucoma 2017;26:303-310. DOI: 10.1097/IJG.0000000000000365.

22. Djordjević-Jocić J, Zlatanovic G, et al. Transforming growth factor beta1, matrix-metalloproteinase-2 and its tissue inhibitor in patients with pseudoexfoliation glaucoma/syndrome. Vojnosanit Pregl 2012;69:231-236. DOI: 10.2298/VSP1203231D.

23. Sherwood MB, Grierson I, et al. Long-term morphologic effects of antiglaucoma drugs on the conjunctiva and Tenon's capsule in glaucomatous patients. Ophthalmology 1989;96:327-335. DOI: 10.1016/S0161-6420(89)32888-0.

24. Takihara $Y$, Inatani $M$, et al. Trabeculectomy for open-angle glaucoma in phakic eyes vs in pseudophakic eyes after phacoemulsification: a prospective clinical cohort study. Jama Ophthalmol 2014;132:69-76. DOI: 10.1001/jamaophthalmol.2013.5605.

25. Fukuchi T, Ueda J, et al. The outcome of mitomycin C trabeculectomy and laser suture lysis depends on postoperative management. Jpn J Ophthalmol 2006;50:455-459. DOI: 10.1007/s10384-006-0346-9.

26. Cho HK, Kojima S, et al. Effect of laser suture lysis on filtration openings: a prospective three-dimensional anterior segment optical coherence tomography study. Eye 2015;29:1220-1225. DOI: 10.1038/ eye.2015.129.

27. Ralli M, Nouri-Mahdavi K, et al. Outcomes of laser suture lysis after initial trabeculectomy with adjunctive mitomycin C. J Glaucoma 2006;15:60-67. DOI: 10.1097/01.ijg.0000195929.94922.a2.

28. Kapetansky FM. Laser suture lysis after trabeculectomy. J Glaucoma 2003;12:316-320. DOI: 10.1097/00061198-200308000-00005.

29. Kromer M, Nolle B, et al. Laser suture lysis after trabeculectomy with mitomycin C: analysis of suture selection. J Glaucoma 2015;24:e84-87. DOI: 10.1097/IJG.0000000000000144.

30. Tojo N, Otsuka M, et al. Conventional trabeculectomy vs trabeculectomy with the $\operatorname{Ex}-\operatorname{PRESS}((\mathrm{R}))$ mini-glaucoma shunt: differences in postoperative interventions. Clin Ophthalmol 2018;12:643-650. DOI: 10.2147/OPTH.S160342.

31. Haga $A$, Inatani $M$, et al. Risk factors for choroidal detachment after trabeculectomy with mitomycin C. Clin Ophthalmol 2013;7:1417-1421. DOI: 10.2147/OPTH.S46375.

32. Altan C, Ozturker C, et al. Post-trabeculectomy choroidal detachment: not an adverse prognostic sign for either visual acuity or surgical success. Eur J Ophthalmol 2008;18:771-777. DOI: $10.1177 / 112067210801800518$.

33. De Feo F, Bagnis A, et al. Efficacy and safety of a steel drainage device implanted under a scleral flap. Can J Ophthalmol 2009;44:457-462. DOI: 10.3129/i09-120. 
34. Varadaraj V, Sengupta S, et al. Evaluation of Angle Closure as a Risk Factor for Reduced Corneal Endothelial Cell Density. J Glaucoma 2017;26:566-570. DOI: 10.1097/IJG.0000000000000669.

35. Law SK, Nguyen AM, et al. Severe loss of central vision in patients with advanced glaucoma undergoing trabeculectomy. Arch Ophthalmol 2007;125:1044-1050. DOI: 10.1001/archopht.125.8.1044.

36. Costa VP, Smith M, et al. Loss of visual acuity after trabeculectomy. Ophthalmology 1993;100:599-612. DOI: 10.1016/S0161-6420(93) 31597-6.
37. Benson SE, Mandal K, et al. Is post-trabeculectomy hypotony a risk factor for subsequent failure? A case control study. BMC Ophthalmol 2005;5:7. DOI: 10.1186/1471-2415-5-7.

38. Tseng VL, Kim CH, etal.RiskFactors and Long-Term Outcomes in Patients with Low Intraocular Pressure after Trabeculectomy. Ophthalmology 2017;124:1457-1465. DOI: 10.1016/j.ophtha.2017.05.014.

39. Bardak Y, Cuypers MH, et al. Ocular hypotony after laser suture lysis following trabeculectomy with mitomycin C. Int Ophthalmol 1997;21:325-330. DOI: 10.1023/A:1006024522541. 